\title{
Artéria renal dupla em gato
}

\section{Double renal artery in cat}

\author{
Fernanda Marques Pestana ${ }^{1}$; Magno dos Santos Roza'; ${ }^{2}$ José Miguel Farias \\ Hernandez $^{3}$; Bárbara Xavier Silva ${ }^{4}$; Marcelo Abidu-Figueiredo ${ }^{5 *}$
}

\begin{abstract}
Resumo
O conhecimento das variações nos vasos renais possui importância em um programa de sistematização da anatomia radiológica e cirúrgica, tanto para o homem quanto para animais destinados a pesquisa, ensino e treinamento cirúrgico. As artérias renais têm sido estudas tanto radiograficamente quanto através de dissecção. Atenção particular é dada ao estudo desses vasos enfatizando as variações entre as diferentes espécies animais. O local de origem das artérias renais a partir da aorta abdominal varia de acordo com a topografia renal nos diferentes animais. A artéria renal direita se origina mais cranialmente que a esquerda de acordo com a posição mais cranial do rim direito. O objetivo deste artigo é descrever um caso de dupla artéria renal esquerda originando-se da superfície ventral da artéria aorta abdominal em um cadáver de gato macho com três anos de idade. O mesmo foi fixado e preservado com solução de formaldeído a $10 \%$ e teve o seu sistema arterial preenchido com látex corado. Observou-se que o rim esquerdo foi suprido por duas artérias renais, de diferente topografia e arranjo, caracterizando duplicidade da artéria renal.
\end{abstract}

Palavras-chave: Duplicidade, artéria renal, gato

\begin{abstract}
Knowledge of the renal vessels variations has importance in a systematization program of radiological and surgical anatomy, both in humans and animals, applied for research and surgical training. Renal arteries have been considered by dissective or angiographic study means. Particular attention has been paid to the study of these vessels, outlining the variations noticed among various animal species. The renal arteries sites of origin of the abdominal aorta vary according to the renal topography of the different animals. As a rule, the right artery arises more cranially than the left one, according with the most cranial position of the right kidney. Thus, the goal of this article is to describe a case of a left double renal artery originating from the ventral portion of the aorta, in a three old male cat cadaver, formalin-preserved at $10 \%$ and with latex colored vascular injection. It was observed that the left kidney was supplied by two arteries of different topography and arrangements, showing duplicity of the renal artery.
\end{abstract}

Key words: Double, renal artery, cat

\footnotetext{
${ }^{1}$ Médica Veterinária. Mestre em Medicina Veterinária, Patologia e Ciências Clinicas, Universidade Federal Rural do Rio de Janeiro, UFRRJ, Prof ${ }^{a}$ Substituta da Área de Anatomia Animal UFRRJ. E-mail: pestana@ufrrj.br

${ }^{2}$ Aluno de Graduação em Zootecnia, Universidade Federal Rural do Rio de Janeiro, UFRRJ. E-mail: magno2sr@hotmail.com

${ }^{3}$ Biólogo. Professor Substituto da Área de Anatomia Animal Universidade Federal Rural do Rio de Janeiro, UFRRJ. E-mail: jmfh01@yahoo.com.br

${ }^{4}$ Médica Veterinária Autônoma. Mestre em Medicina Veterinária, Patologia e Ciências Clinicas - Universidade Federal Rural do Rio de Janeiro, UFRRJ. E-mail: babi.xavier@gmail.com

${ }^{5}$ Médico Veterinário. Prof. Adjunto da Área de Anatomia Animal, Universidade Federal Rural do Rio de Janeiro, UFRRJ. E-mail: marceloabidu@gmail.com

* Autor para correspondência
} 


\section{Introdução}

Os avanços atuais na cirurgia urológica bem como nos procedimentos intervencionistas radiológicos têm apontado para um grande interesse em anatomia vascular renal, pois o completo entendimento desta anatomia é essencial para a realização segura e eficaz desses procedimentos (KHAMANARONG et al., 2004).

No homem as variações da anatomia vascular renal foram caracterizados por diferentes autores, bem como a prevalência de vasos múltiplos que ocorre em cerca de $20-50 \%$ dos casos (TESTUT; LATARJET, 1959; ISODA et al., 2002; BORDEI; SAPTE; ILIESCU, 2004; SAMPAIO, 2007).

Segundo Harrison, Flye e Seigler (1978), Awojobi, Ogunbiyi e Nkposong (1983), Kinnumen et al. (1985) e Sampaio (2007) a multiplicidade da artéria renal, é mais freqüente que a ocorrência de variações numéricas venosas. Segundo vários autores, as variações das artérias renais são carcterizadas por duas ou três artérias que se originam diretamente da artéria aorta abdominal (POIRIER; CHARPY, 1920; TESTUT; LATARJET, 1959; BORDEI; SAPTE; ILIESCU, 2004; SAMPAIO, 2007).

Nos animais domésticos os rins normalmente estão posicionados na região lombar entre as últimas vértebras torácicas e primeiras lombares e recebem o suprimento sanguíneo de ramos primários da artéria aorta abdominal, as artérias renais direita e esquerda, ambas com origem nas superfícies laterais deste vaso. Estas artérias recebem aproximadamente $20 \%$ do fluxo sanguíneo da artéria aorta abdominal (KÖNIG; MAIERL; LIEBICH, 2004). Em função da localização cranial do rim direito quando comparado ao esquerdo, a artéria renal direta também tende a ser cranial à esquerda, embora seja possível a origem de ambas no mesmo nível (NICKEL; SCHUMMER; SEIFERLE, 1979; 1981).

No gato doméstico, as artérias renais originamse assimetricamente das superfícies laterais da artéria aorta abdominal, ao nível da primeira vértebra lombar (GETTY, 1986) ou entre a terceira e a quarta (NICKEL; SCHUMMER; SEIFERLE, 1979). Segundo Gorham e Tower (1895) há a possibilidade de duplicidade das artérias no gato, embora não informe a freqüência, esqueletopia e em qual antímero esta variação é mais freqüente.

O objetivo deste artigo é relatar um caso de duplicidade da artéria renal esquerda em gato, com enfoque nas possibilidades de implicação clínicocirúrgica desta variação anatômica.

\section{Relato de Caso}

No decorrer das atividades práticas de dissecção realizadas nas disciplinas do Laboratório de Anatomia Animal do Departamento de Biologia Animal da Universidade Federal Rural do Rio de Janeiro (UFRRJ), observou-se uma variação na origem da artéria renal esquerda de um gato de aproximadamente três anos de idade, mestiço, macho que foi fixado com solução de formaldeído a $10 \%$ e teve o seu sistema arterial preenchido com solução aquosa de Petrolátex corado. $\mathrm{O}$ animal foi dissecado rebatendo-se as vísceras abdominais para a evidenciação do pedículo renal esquerdo, origem, trajeto e esqueletopia das artérias renais. Com um paquímetro de precisão foram obtidas medidas do comprimento, largura e espessura do rim esquerdo.

O rim esquerdo apresentou $3,9 \mathrm{~cm}$ de comprimento, 2,6 cm de largura e $2,8 \mathrm{~cm}$ de espessura. Observou-se que o rim esquerdo apresentou duas artérias renais, uma cranial e outra caudal. A primeira artéria se originou ventralmente à porção abdominal da aorta de forma única em nível da terceira vértebra lombar (L3) medindo 0,9 $\mathrm{cm}$ de comprimento e bifurcou em 2 ramos (Figura 1): um ramo que se dirigiu diretamente para o hilo renal medindo $1,4 \mathrm{~cm}$ de comprimento, emitindo ainda um ramo para a glândula adrenal medindo $1,6 \mathrm{~cm}$ de comprimento; o segundo ramo medindo $1,3 \mathrm{~cm}$ de comprimento seguindo diretamente para o hilo renal. A segunda artéria renal emergiu 
lateralmente à porção abdominal da aorta de forma única em nível da terceira vértebra lombar (L3) medindo $1,4 \mathrm{~cm}$ de comprimento também seguindo diretamente para o hilo renal. $\mathrm{O}$ rim direito também foi dissecado, entretanto não apresentou nenhuma variação vascular, apesar de estar visivelmente assimétrico em relação ao tamanho do esquerdo (Figura 2).

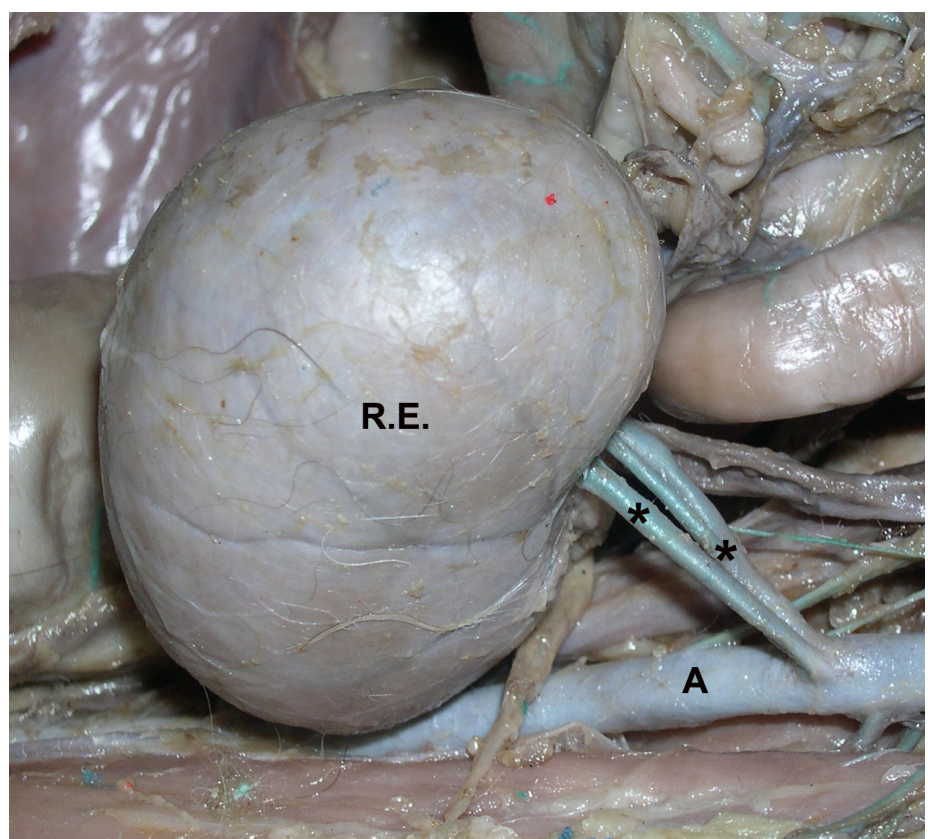

Figura 1. Fotomacrografia do rim esquerdo mostrando a variação numérica da artéria renal. $\mathrm{A}=$ Porção abdominal da artéria aorta $\quad$ R.E. $=$ Rim esquerdo $\quad *=$ Artérias renais múltiplas

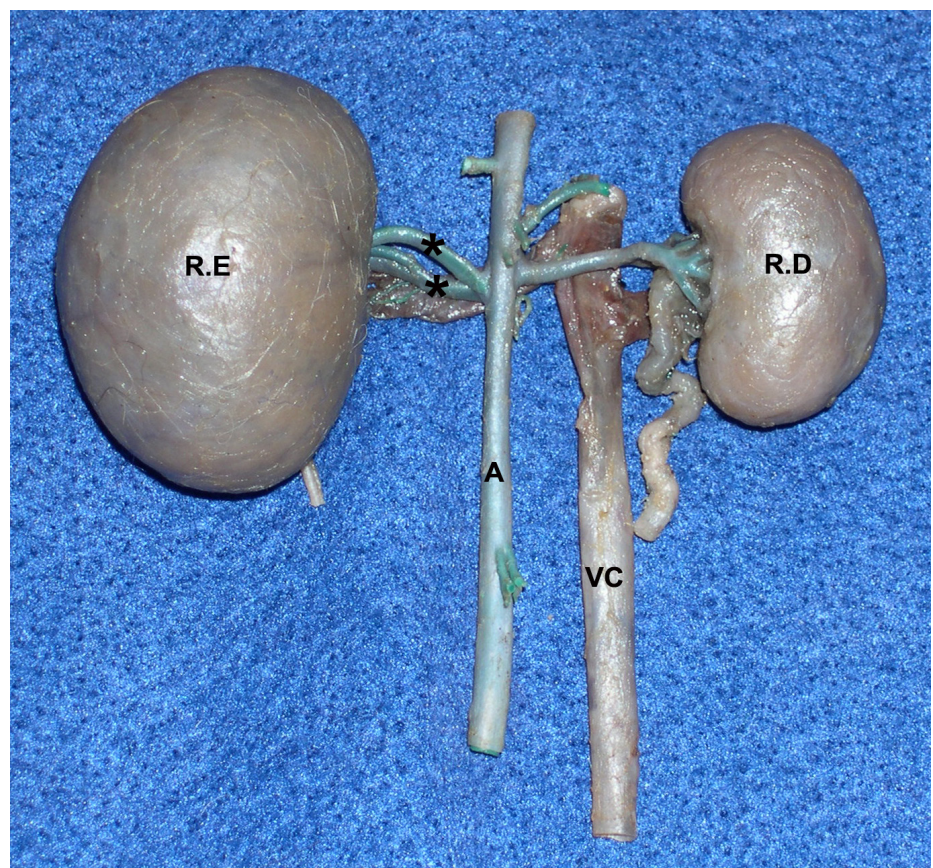

Figura 2. Fotomacrografia da vista dorsal dos rins mostrando a variação numérica da artéria renal esquerda. $\mathrm{A}=$ Porção abdominal da artéria aorta $\quad$ R.D $=$ Rim direito $\quad$ R.E $=$ Rim esquerdo $\mathrm{VC}=$ Veia cava caudal $\quad *=$ Artérias renais múltiplas 


\section{Discussão}

Qualquer flexibilidade na morfologia e topografia de estruturas do corpo que não altere sua fisiologia pode ser considerada uma variação anatômica e, portanto, deve ser diferenciada de aberrações e má formações, pois nestas há influência negativa sob o ponto de vista fisiológico (SYKES, 1963; SANUDO; VAZQUEZ; PUERTA, 2003). No entanto, as variações podem estar correlacionadas com predisposições à certas enfermidades e achados em exames clínicos (WILLAM, HUMPHERSON, 1999).

Mais recentemente as artérias renais foram descritas em alguns mamíferos, tais como camelos, caprinos, ovinos, suínos e javalis, embora não tenha sido observada duplicidade (QIUSHENG, 2000; ASLAN; NAZLI, 2001; MARDANI; AHMADIGHOJABAIGLO， 2001; VODENICHAROV; DANCHEV, 2003). A ocorrência e descrição das artérias renais duplas são escassas na medicina veterinária. Em jumentos nordestinos as artérias renais duplas foram encontradas em 3 de uma amostragem de 30 animais, sempre no rim esquerdo (SILVA NETO; PEDUTI NETO, 1995). Wiland e Indykiewicz (1999) relataram a ocorrência de artérias renais duplas em cães e visons, ressaltando a prevalência desta variação no antímero esquerdo, contudo, o fato que mais chama atenção foi a duplicicade destes vasos nos dois antímeros, em uma cadela. Alonso e Abidu-Figueiredo (2008) e Oliveira e Guimarães (2007) relataram a ocorrência de duplicação das artérias renais esquerda e direita em cão, respectivamente.

A origem das artérias renais esquerdas duplas a partir da superfície ventral da artéria aorta abdominal contrasta com as informações fornecidas por outros autores, que mencionam ser lateral a origem destes vasos em diferentes animais (NICKEL; SCHUMMER; SEIFERLE, 1979, 1981; JAIN; SINGH, 1987).

Em humanos, Karmacharya et al. (2006) citam a prevalência de artérias renais acessórias no rim esquerdo. As variações no arranjo e distribuição das artérias renais também foram estudadas por Sampaio e Passos (1992), que citaram casos de duplicação e triplicação, e até mesmo a ocorrência de ramos arteriais suprindo tanto o pólo superior quanto o pólo inferior do rim. Nestes casos, os autores sugeriram a adoção do termo "artérias renais múltiplas", por se tratarem de segmentos terminais normais, sem anastomoses entre eles e, sob o ponto de vista fisiológico, tão importantes para o suprimento sanguíneo renal. Os mesmos autores reforçam a importância da adequação na nomenclatura empregada para tais vasos e atentam para os termos "aberrante", "acessório", "extra", e "supranumerário", pois transmitem a falsa idéia de serem elementos de pouca importância.

Ainda fazendo referência ao homem, a presença de artérias renais múltiplas aumenta a complexidade do transplante renal (FOX; YALIN, 1979), assim como há maior correlação com patologias renais, em comparação aos órgãos supridos por artéria renal única (SAMPAIO; PASSOS, 1992). Khamanarong et al. (2004) destacam a importância do entendimento da anatomia vascular renal na eficiência e segurança de procedimentos cirúrgicos e radiológicos.

Sob ponto de vista mais geral, qualquer cirurgia abdominal que necessite de mobilização ou controle hemostático da artéria renal esquerda e suas ramificações requerem uma busca sistemática por eventuais variações anatômicas vasculares. A complexidade destas variações vasculares pode modificar as possibilidades técnicas do procedimento cirúrgico.

Variações numéricas bem como, na origem das artérias renais em gatos devem ser consideradas na execução dos procedimentos radiológicos, cirúrgicos e experimentais visando dessa maneira, evitar que erros sejam cometidos por desconhecimento das possibilidades da existência de artérias renais duplas nestes animais. 


\section{Referências}

ALONSO, L. S.; ABIDU-FIGUEIREDO, M. Artéria renal dupla originando da aorta em cão: relato de caso, Semina: Ciências Agrárias, Londrina, v. 29, n. 1, p.185188, 2008.

ASLAN, K.; NAZLI, M. A comparative macro-anatomic investigation on the intrarenal segmentation of the renal artery in goats and Morkaraman sheep. Ind. Vet. J., Chennai, India, v. 78, n. 2, p. 139-143, 2001.

AWOJOBI, O. A.; OGUNBIYI, O. A.; NKPOSONG, E. O. Unusual relationship of multiple renal arteries. Urology, Cleveland, v. 21, n. 2, p. 205-6, 1983.

BORDEI, P.; SAPTE, E.; ILIESCU, D. Double renal arteries originating from the aorta. Surg. Radiol. Anat., Berlin, v. 26, n. 6, p. 474-9, 2004.

FOX, M.; YALIN, R. Renal transplantation with multiple arteries. British Journal of Urology, Londres, v. 51, n. 5, p. 333-336, 1979.

GETTY, R. Anatomia dos animais domésticos. Rio de Janeiro: Guanabara Koogan, 1986. 1136 p.

GORHAM, F. P.; TOWER, R. W. A laboratory guide for dissection of the cat. In: GORHAM, F. P. Vascular system. New York: Henry Holt Editora, 1895. p. 356-360.

HARRISON JUNIOR, L. H.; FLYE, M. W.; SEIGLER, H. F. Incidence of anatomical variants in renal vasculature in the presence of normal renal function. Ann. Surg., Filadélfia, v. 188, n. 11, p. 83-89, 1978.

ISODA, H.; SAITOH, M.; ASAKURA, T.; AKAI, M.; ITAGAKI, Y.; HA- KAWA, S. K.; HARIMA, K.; SAWADA, S. An unusual arterial supply of the kidney from the opposite renal artery. Comput. Med. Imaging. Graph., Nova Yorque, v. 26, n. 5, p. 353-355, 2002.

JAIN, R. K.; SINGH, Y. Vascularization of kidneys in bovine. Indian Veterinary Journal, Chennai, India, v. 64, n. 3, p. 1059-1062, 1987.

KHAMANARONG, K.; PRACHANEY, P.; UTRARAVICHIEN, A.; TONG-UN, T.; SRIPAORAYA, K. Anatomy of renal arterial supply. Clin. Anal., Chicago, v. 17, n. 4, p. 334-336, 2004.

KARMACHARYA, J.; PARMER, S. S.; ANTEZANA, J. N.; FAIRMAN, R. M.; WOO, E. Y.; VELAZQUEZ, O. C.; GOLDEN, M. A.; CARPENTER, J. P. Outcomes of accessory renal artery occlusion during endovascular aneurysm repair. J Vasc Surg., Londres, v. 43, n. 1, p. 8-12, 2006.
KÖNIG, H. E.; MAIERL, J.; LIEBICH, H. G. Órgãos urinários (organa urinaria). In: KÖNIG, H. E.; LIEBICH, H. G. Anatomia dos animais domésticos: texto e atlas colorido. Porto Alegre: Artmed, 2004. p. 103-118.

MARDANI, K.; AHMADI-GHOJABAIGLO, A. Branching pattern of the renal artery and urinary collecting system of the sheep kidney, using their cast by latex and mica. Iran J. Vet. Res., Shiraz, v. 2, n. 2, p. 182-186, 2001.

NICKEL, R.; SCHUMMER, A.; SEIFERLE, E. The viscera of domestic mammals. Berlin: Verlag Paul Parey, 1979. $400 \mathrm{p}$.

The anatomy of the domestic animals. Berlin: Verlag Paul Parey, 1981. 610 p.

OLIVEIRA, F. S.; GUIMARÃES, G. C. Duplicidade da artéria renal em cão. Cienc. Rural, Santa Maria, v. 37, n. 6, p. 1817-1819, 2007.

POIRIER, P.; CHARPY, A. Traité d'anatomie humaine. In: . Angeliologie. Paris: Masson, 1920. v. 5, p. $88-110$

QIUSHENG, C.; YI, L. Renal artery distribution in kidneys of bactrian camels. Chin J Vet Sci., China, v. 20, n. 6 , p. 565-568, 2000.

SAMPAIO, F. J. B. Anatomia renal para urologia. In: Anatomia vascular do rim. Rio de Janeiro:

Gráfica e Editora Prensa, 2007. p. 73-81.

SAMPAIO, F. J. B.; PASSOS, M. A. R. F. Renal arteries: anatomic study for surgical and radiological practice. Surgical and Radiologic Anatomy, Berlin, v. 14, n. 2, p. 113-117, 1992.

SANUDO, J.; VAZQUEZ, R.; PUERTA J. Meaning and clinical interest of the anatomical variations in the $21 \mathrm{st}$ century. Eur J Anat., Salamanca, v. 7, n. 1, p. 1-3, 2003.

SILVA NETO, E. J. da; PEDUTI NETO, J. Número e distribuição dos elementos vasculares, arteriais e venosos, no hilo renal de jumentos nordestinos (Asinus asinus, Gray 1824, ex. Fritsch 1775). Revista da FZVA Uruguaiana, Uruguaiana, v. 2/3, n. 1, p. 41-61, 1995/1996.

SYKES, D. The arterial supply of the human kidney with special reference to accessory renal arteries. Br J Surg., Inglaterra, v. 50, p. 368-374, 1963.

TESTUT, L.; LATARJET, A. Tratado de anatomía humana. In: . Sistema urinário. Barcelona: Salvat, 1959. p. 871-87. 
VODENICHAROV, A.; DANCHEV, S. Comparative anatomical study of renal artery architechtonics in the wild pig Sus scrofa scrofa and the domestic pig Sus scrofa domestica. Bulg J. Vet. Med., Zagora, v. 6, n. 1, p. 1-8, 2003.

WILAND, C.; INDYKIEWICZ, P. Multiple renal arteries (Aa. renales) in mink and dog. Electronic Journal of
Polish Agricultural Universities, Polônia, v. 2, n. 2, p. 12-17, 1999.

WILLAM, P. L.; HUMPHERSON, J. R. Concepts of variation and normality in morphology: important issues at risk of neglect in modern undergraduate medical courses. Clin Anat., Chicago, v. 12, n. 3, p. 186-190, 1999. 